\title{
THE LHC BEAM PIPE WAVEGUIDE MODE REFLECTOMETER
}

\author{
T.Kroyer, P. Borowiec ${ }^{1)}$, F. Caspers, T. Kroyer, Z. Sulek ${ }^{1)}$, L.R. Williams \\ CERN, Geneva, Switzerland
}

\begin{abstract}
The waveguide-mode reflectometer for obstacle detection in the LHC beam pipe has been intensively used for more than 18 months. The "Assembly" version is based on the synthetic pulse method using a modern vector network analyzer. It has mode selective excitation couplers for the first TE and TM mode and uses a specially developed waveguide mode dispersion compensation algorithm with external software. In addition there is a similar "In Situ" version of the reflectometer which uses permanently installed microwave couplers at the end of each of the nearly $3 \mathrm{~km}$ long LHC arcs. During installation a considerable number of unexpected objects have been found in the beam pipes and subsequently removed. Operational statistics and lessons learned are presented and the overall performance is discussed.
\end{abstract}

${ }^{1)}$ on leave from HNINP, Krakow, Poland

CERN, 


\title{
THE LHC BEAM PIPE WAVEGUIDE MODE REFLECTOMETER
}

\author{
T. Kroyer, P. Borowiec ${ }^{\dagger}$, F. Caspers, Z. Sulek ${ }^{\dagger}$, L. R. Williams, CERN, Geneva, Switzerland
}

\section{Abstract}

The waveguide-mode reflectometer for obstacle detection in the LHC beam pipe has been intensively used for more than 18 months. The "Assembly" version is based on the synthetic pulse method using a modern vector network analyzer. It has mode selective excitation couplers for the first TE and TM mode and uses a specially developed waveguide mode dispersion compensation algorithm with external software. In addition there is a similar "In Situ" version of the reflectometer which uses permanently installed microwave couplers at the end of each of the nearly $3 \mathrm{~km}$ long LHC arcs. During installation a considerable number of unexpected objects have been found in the beam pipes and subsequently removed. Operational statistics and lessons learned are presented and the overall performance is discussed.

\section{INTRODUCTION}

During start-up and commissioning of several accelerators unwanted objects were found in the beam pipe. One very well known example were bottles found 10 years ago in the LEP beam pipe. In CERN's LHC even much smaller obstacles would cause major problems, since as a cryogenic machine LHC is very sensitive to particle losses. Recent energy deposition simulations showed that particles scattered even by very thin objects ( $0.1 \mathrm{~mm}$ thickness) can rapidly quench a downstream magnet [1]. Quenches can already be initiated by the pilot beam, i.e. the lowest intensity beam foreseen for LHC commissioning (a single bunch with $5 \cdot 10^{9}$ protons), and they can happen before the obstacle is vaporized.

The waveguide mode reflectometer was developed as part of the quality assurance program for LHC to detect anomalies in the LHC cold arcs. It exists in two distinct versions:

- The Assembly Version, which was used for inspection of single magnets and chains of interconnected magnet during the installation of the machine.

- The In Situ Version, which relies on couplers permanently installed in the machine for measurements during start-up and operation.

For both options special mode selective couplers were developed. The measurements are made on a vector network analyser in the frequency domain. After external signal processing, including the numerical removal of the waveguide dispersion, the reflection pattern on the line is plot-

\footnotetext{
${ }^{\dagger}$ On leave from HNINP, Kraków, Poland
}

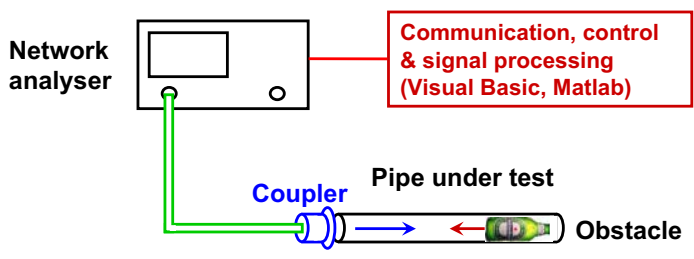

Figure 1: System overview of the LHC reflectometer.

ted and analysed. The measurement set-up is sketched in Fig. 1. For a more detailed description of the system the reader is referred to [2].

\section{ASSEMBLY VERSION}

Since November 2005 the magnets have been checked systematically in the LHC tunnel by visual inspection and using microwave reflectometry. By June 2006 a number of suspicious cases had turned up among the 400 magnets inspected and five metallic obstacles had been removed [3]. It was thus decided to considerably tighten the quality assurance measures [4]. The suspicious cases were reexamined by endoscopy and 14 more objects recovered, in some cases necessitating the reopening of interconnection modules that had already been installed. The majority of objects were metal swarfs projecting inwards from the welding strip inside the beam screen. They originated from the manufacturing process and had survived all standard cleaning procedures applied at CERN. Consequently, after this finding all beam screens were brushed mechanically before the usual bath cleaning.

Additionally, all subsequent magnets were checked with reflectometry as well as by visual means and endoscopy after assembly in a surface building, where visual inspection and any kind of intervention are easier. Thereafter the beam screen extremities were sealed before the transport of the magnet to its final position in the tunnel, not to be reopened until their interconnection with the next magnet. This procedure substantially decreased the number of objects reducing the beam aperture, as was found during final checks on chains of interconnected magnets. Table 1 summarizes the results of magnet inspections before and after the revision of the quality assurance procedures.

In order to integrate the final reflectometry checks into the general installation procedure they were mostly made over two-cell sections (16 interconnected magnets, total length $\approx 216 \mathrm{~m}$ ) accessible from both sides. Due to the extended length the noise floor is higher than for single magnets, 
Table 1: Overview of the objects identified in and retrieved from magnet beam pipes during the stages of the installation. The quality assurance (QA) procedures were tightened after numerous findings in the beginning of LHC installation. By visual inspection and endoscopy on the surface in about $20 \%$ of the magnets dust contamination, small plastic pieces etc. were found in the beam line.

\begin{tabular}{c|c|c|c} 
QA Procedure & location & \# magnets & \# obstacles \\
\hline \hline Initial & tunnel & 400 & 19 \\
\hline Revised, reflec. & surface & 900 & 19 \\
\hline Final insp. & tunnel & 1000 & 2
\end{tabular}

which results in a reduction of sensitivity especially in the range above 50 to $100 \mathrm{~m}$. By June 2007, with most magnets interconnected, two objects have been found during final inspection.

Fig. 2(a) depicts a typical case where an object was localized in the fourth magnet in a chain of 16. In addition to the interconnection modules a number of spurious peaks can be seen in the traces, most of which are due to multiple reflections. Interconnect 2 is at the upstream end of a quadrupole, where the beam position monitors (BPM) are installed. Since these pick-ups couple to the $\mathrm{TM}_{01}$ waveguide mode, part of the signal runs along the BPM cables and gets reflected at the end that was open at the time of the measurement. As a result peaks spaced by about $1.2 \mathrm{~m}$ appear (Multiple reflections 1 in Fig. 2(a)). A second source of spurious peaks are multiple reflections between interconnects, e.g. Multiple reflection 2, which comes from Interconnect 1 and Interconnect 2 . There are ways to identify such spurious reflections [2], but a loss of sensitivity is unavoidable.

Due to the large distance to the end of the chain one of the interconnection modules had to be cut to remove the object, which turned out to be a piece of aluminium foil trapped in a pumping slot (Fig. 2(b)). This piece probably comes from aluminium foil used temporarily to close the beam screen extremities to protect them from other contaminations. The second object found during final inspection is depicted in Fig. 3 and has been identified as a piece of contact spring from the beam line interconnection modules.

Experience showed that it is not easy to estimate the size of an object from the amplitude of a peak in the reflection signal. This is due to the fact that the field distortion caused by an object depends on its orientation and material parameters. Metallic objects projecting inwards from the beam screen walls give high signals, metallic ones oriented parallel to the wall and dielectric ones without any orientation give low signals. Since the $\mathrm{TM}_{01}$ and the fundamental TE mode used have different field patterns, a given object has different signatures with these two modes. In several cases it was possible get indications of the properties of an object from data derived from the two modes.

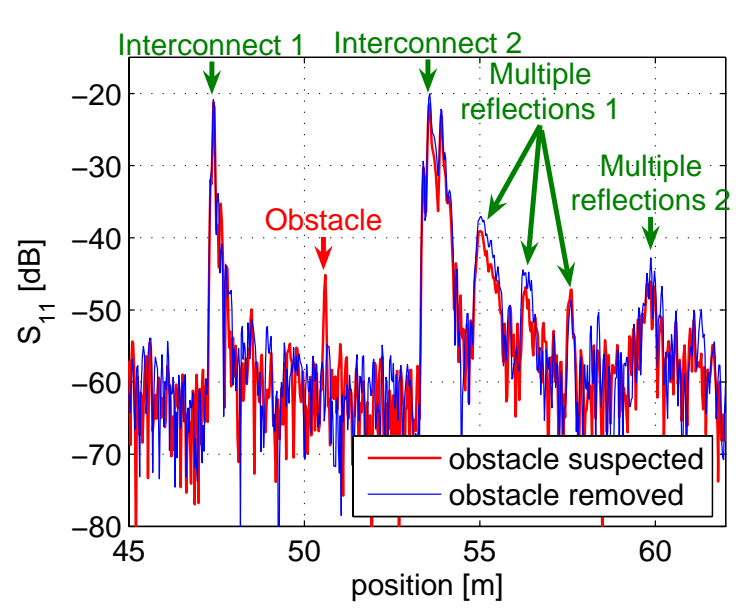

(a) The unexpected peak inside a quadrupole was identified as an obstacle (red trace). Shown is data from the $\mathrm{TM}_{01}$ mode; indications were also seen in TE mode data. The peaks after interconnect 2 are due to various multiple reflections.

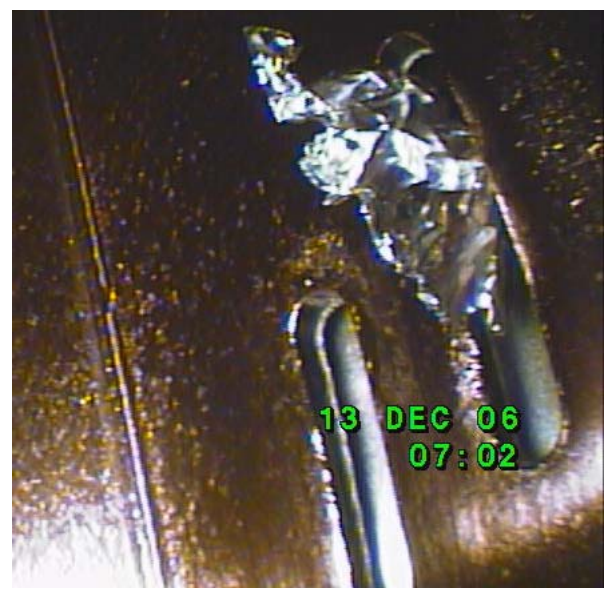

(b) Picture of the obstacle taken with an endoscope before it was removed. The pumping slot length is about $10 \mathrm{~mm}$.

Figure 2: An obstacle found during final inspection in the fourth magnet of a chain of 16 interconnected magnets.

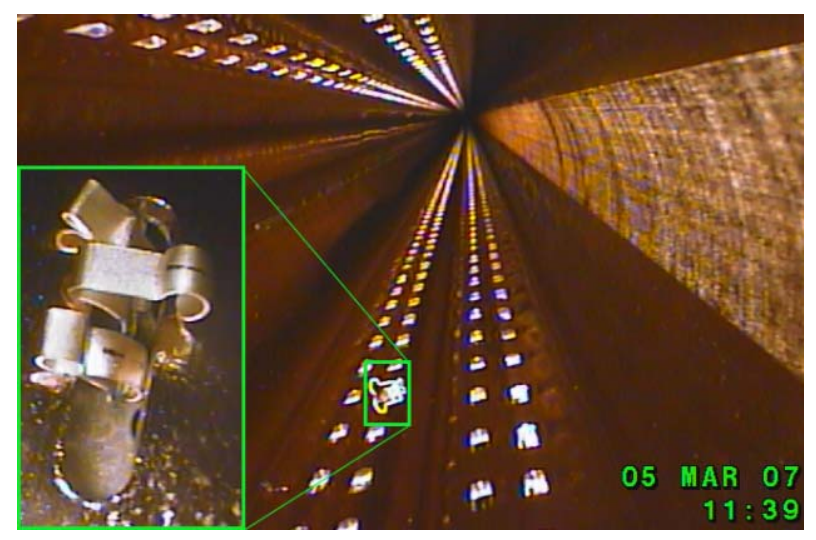

Figure 3: Obstacle found during final inspection in the third magnet in a chain of 16 . 


\section{IN SITU VERSION}

The In Situ Version of the reflectometer is based on permanently installed couplers at the end of each $2800 \mathrm{~m}$ long arc [2]. At cryogenic temperature the waveguide attenuation should be low enough to allow reflection measurements over half an octant. However, due to various restrictions on the coupler design the expected sensitivity will not be as good as that of the Assembly Version. Therefore it is planned to make relative measurements by taking reference data for each octant once it is cooled down. In case a problem appears later, new measurements can be made and compared to the reference data to find indications about what has changed. In preliminary measurements numerous multiple reflections from the interconnects were found that may pose difficulties, in particular since the interconnects move during cool-down and warm-up of the machine, which might change their electric properties of each interconnect.

\section{Transmission Measurement}

In order to check the properties of the installed couplers and to verify estimations of the line attenuation, a transmission measurement was made along a full octant at cryogenic temperatures. A standard RF signal generator was used as transmitter and a spectrum analyser as receiver. In both beam screens three bands with good transmission properties were found within the coupler pass-bands (Fig. 4). The transmission response is very ragged, which is very likely due to the frequency-selective scattering of the interconnection modules. The minimum attenuation found was $0.039 \mathrm{~dB} / \mathrm{m}$ at 6.1 and $7.8 \mathrm{GHz}$, which is somewhat higher than estimated in [2]. The transmission measurement could be used to observe changes in attenuation with time and for example as a function of the static magnetic field due to the magneto-resistive effect.

\section{CONCLUSION}

The Assembly Version of the reflectometer has been used together with visual checks for object detection as part of the quality assurance program for the LHC beam screen during the magnet installation and interconnection process. In the first months of operation several objects were identified in magnets in the tunnel, most of them remainders from the beam screen production process. As a consequence the beam screen cleaning and inspection procedures were improved: magnets were preinspected visually and by reflectometry on the surface; a final inspection by reflectometry was then performed on $200 \mathrm{~m}$ long sections of interconnected magnets in the tunnel. This procedure has resulted in a substantial reduction of obstacles found in magnets in the tunnel. However, even though a considerable number of objects were found and even though a large effort was devoted to optimize the quality assurance and inspection, there cannot be certainty that all objects have been found. The In Situ Version works with couplers permanently in-

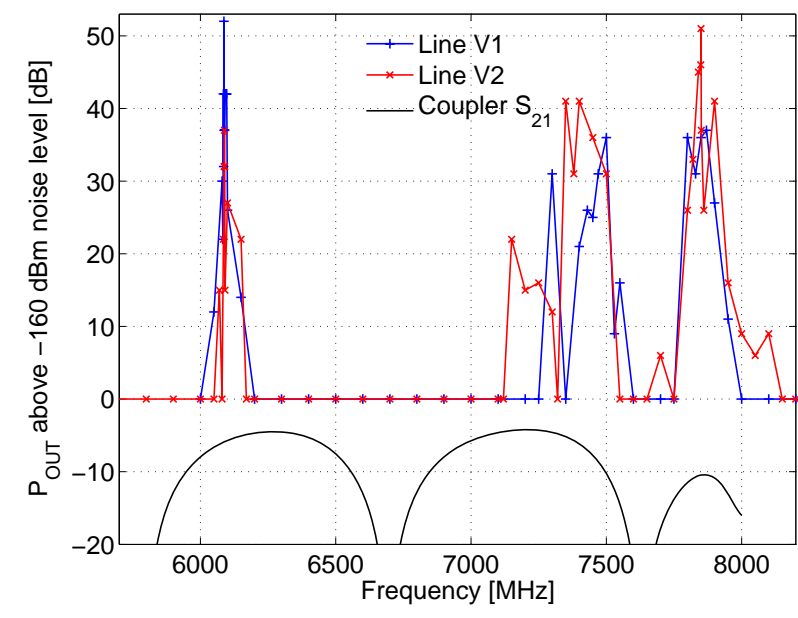

Figure 4: Transmission of a microwave signal inside the beam screen along a $2800 \mathrm{~m}$ long octant at cryogenic temperature. The input power was $+20 \mathrm{dBm}$. The transmission windows correspond well to the simulated coupler response.

stalled at the end of each octant. Due to the large distances and the limitations on the coupler design it is expected to be less sensitive than the Assembly Version. Measurements are planned when the octants are at low temperature to be stored as reference data for comparison in case the need arises. However, temperature cycling of the octant might change the electrical properties of the interconnects. Furthermore, a transmission measurement over an entire $2800 \mathrm{~m}$ long octant at cryogenic temperature was carried out.

\section{ACKNOWLEDGEMENTS}

We would like to thank the AB-RF workshops, Nico Kos, Alexis Vidal, Patrick Coly and Paul Cruikshank for help. Thanks to Elena Shaposhnikova, Flemming Pedersen, Trevor Linnecar and Steve Myers for support and fruitful discussions as well as to Alain Poncet, Philippe Lebrun and Lyn Evans for support. A special thanks goes to the members of the inspection teams, who collected all the data presented here.

\section{REFERENCES}

[1] Brügger, M., Cerutti, F., Ferrari, A., Vlachoudis, V., Fluka Estimations concerning Obstacles in the LHC Magnets, CERN-AB-Note-2007-018 (2007)

[2] Kroyer, T., Application of Waveguide Mode Diagnostics for Remote Sensing in Accelerator Beam Pipes, CERNTHESIS-2005-061 (2005)

[3] Kroyer, T. et al., Operational experience with the LHC waveguide mode reflectometer, CERN-LHC-ProjectReport-907, Geneva (2006)

[4] Brüning, O., Minutes of the 57. LHC Technical and Commissioning Committee (LTC), http://ab-div-co.web.cern.ch/abdiv/Meetings/ltc/ltc_2006-06.html (2007) 